\title{
Gel electrophoresis and hybridization of DNA- functionalized quantum-dots as part of a study of linear quantum-dot chains
}

\author{
Masaru Oda ${ }^{1,2, *}$, Kosuke Sasano ${ }^{2}$, Akira $\mathrm{Nishi}^{2}$, Tamotsu Zako ${ }^{3}$, and Toshiro Tani ${ }^{4}$ \\ ${ }^{1}$ Department of Basic Sciences, Faculty of Engineering, Kyushu Institute of Technology, 1-1 Sensui- \\ cho, Tobata-ku, Kitakyushu, Fukuoka, 804-8550 Japan \\ ${ }^{2}$ Department of Applied Science for Integrated System Engineering, Graduate School of Engineering, \\ Kyushu Institute of Technology, 1-1 Sensui-cho, Tobata-ku, Kitakyushu, Fukuoka, 804-8550 Japan \\ ${ }^{3}$ Department of Chemistry and Biology, Graduate School of Science and Engineering, Ehime \\ University, 2-5, Bunkyo-cho, Matsuyama, Ehime, 790-8577 Japan \\ ${ }^{4}$ Department of Applied Physics, Tokyo University of Agriculture and Technology, Naka-cho 2-24- \\ 16, Kogane-i, Tokyo, 790-8577 Japan
}

\begin{abstract}
We report the results of our investigation of agarose gel electrophoresis and hybridization of mercaptopropionic-acid-capped quantum dots (MPA-capped QDs) conjugated with short single-stranded DNA, as part of a study of closely spaced linear QD chains. The electrophoresis results suggest that migration speed and separation resolution depend on the amount of MPA on the QD surface. The QDs separated by electrophoresis according to DNA valence, i.e., the amount of DNA per particle, were used as building blocks for the synthesis of linear QD oligomers.
\end{abstract}

\section{Introduction}

Closely spaced quantum dot (QD) oligomers, such as dimers and trimers, have attracted attention over the last two decades due to their optical properties, which are influenced by energy transfer and/or electronic coupling among constituent QDs [1-3]. However, QD oligomer optical properties are still not thoroughly understood, in part because it is difficult to fabricate well-defined QD-oligomer structures, especially linear chains composed of three or more QDs. We recently conducted an investigation to improve QD oligomer synthesis using a DNA hybridization technique. Here, we present the results of this investigation.

\section{Experimental}

QD oligomers were fabricated via the following steps. First, CdSe/ZnS core-shell QDs capped with trioctylphosphineoxide were synthesized via pyrolytic decomposition of

\footnotetext{
* Corresponding author: odamasa@mns.kyutech.ac.jp
} 
organometallic compounds [4]. Next, the QDs were rendered water-soluble by ligand exchange with mercaptopropionic acid [5]. The QDs were conjugated with thiolated short single-stranded (ss) DNA (15 mer) in a borate buffer solution ( $\mathrm{pH}$ 8.3). The DNAfunctionalized QDs were separated using agarose gel electrophoresis according to DNA valence $\left(N_{\mathrm{DNA}}\right)$, i.e., the amount of DNA per particle. The QDs for the formation of linear QD-oligomers $\left(N_{\mathrm{DNA}}=1\right.$ or 2 , as shown in Fig. $\left.1(\mathrm{a})\right)$ were extracted from the gel and linked with similarly prepared QDs conjugated with complementary DNA, as illustrated in Fig. 1(b). The structures of the oligomers were characterized by transmission electron microscopy (TEM). Absorption and photoluminescence (PL) spectra were measured.

\section{Results and Discussion}

Figure 1(c) shows a typical PL image of the agarose gel containing the QDs conjugated with ssDNA after electrophoresis. The QDs were separated into several bands. We confirmed that the migration speed decreases as the DNA valence increases, as shown in Fig. 1 (a) [6]. On the other hand, the migration order is reversed and the bandwidth, i.e., separation resolution, is improved as the amount of MPA on the QD surface decreases. Details of the separation by electrophoresis will be described in the presentation.

Figure 1(d) shows typical TEM images of QD-oligomers obtained after hybridization. Even though precise control of the shape and particle-number was not achieved, it was confirmed that closely spaced QD-oligomers could be obtained by the hybridization.

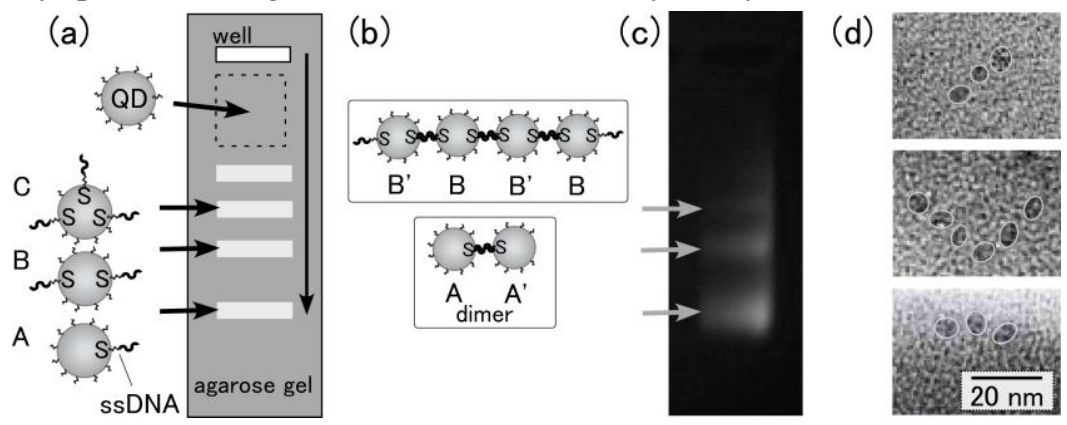

Fig. 1. Schematic diagram of (a) the agarose gel electrophoresis of the DNA-functionalized quantum dots (QDs) (b) QD chains linked by double-stranded DNA. (c) Photoluminescence (PL) image of the agarose gel (4\% NuSieve GTG agarose; Lonza) under 365-nm light irradiation. The molar ratio of the QD to DNA was 1:8. (d) Transmission electron microscopy (TEM) images of QD oligomers. The lines represent the particle outlines.

\section{References}

1. R. Koole, P. Liljeroth, C. Donega, D. Vanmaekelbergh, and A. Meijerink, JACS, 128, 10436 (2006)

2. B. K. Hughes, J. L. Blackburn, D. Kroupa, A. Shabaev, S. Erwin, A. Efros, A. Nozik, J. Luther, and M. Beard, JACS 136, 4670 (2014)

3. G. Tikhomirov, S. Hoogland, P. Lee, A. Fischer, E. Sargent, and S. Kelley, Nature Nanotechnology, 6, 485 (2011)

4. K. Hashizume, M. Matsubayashi, M. Vacha, and T. Tani, J. Lumin., 98, 49 (2002)

5. B. Pong, B. Trout, and J. Lee, Langmuir, 24, 5270 (2008)

6. M. Oda, K. Sasano, A. Nishi, T. Zako, and T. Tani, submitted 